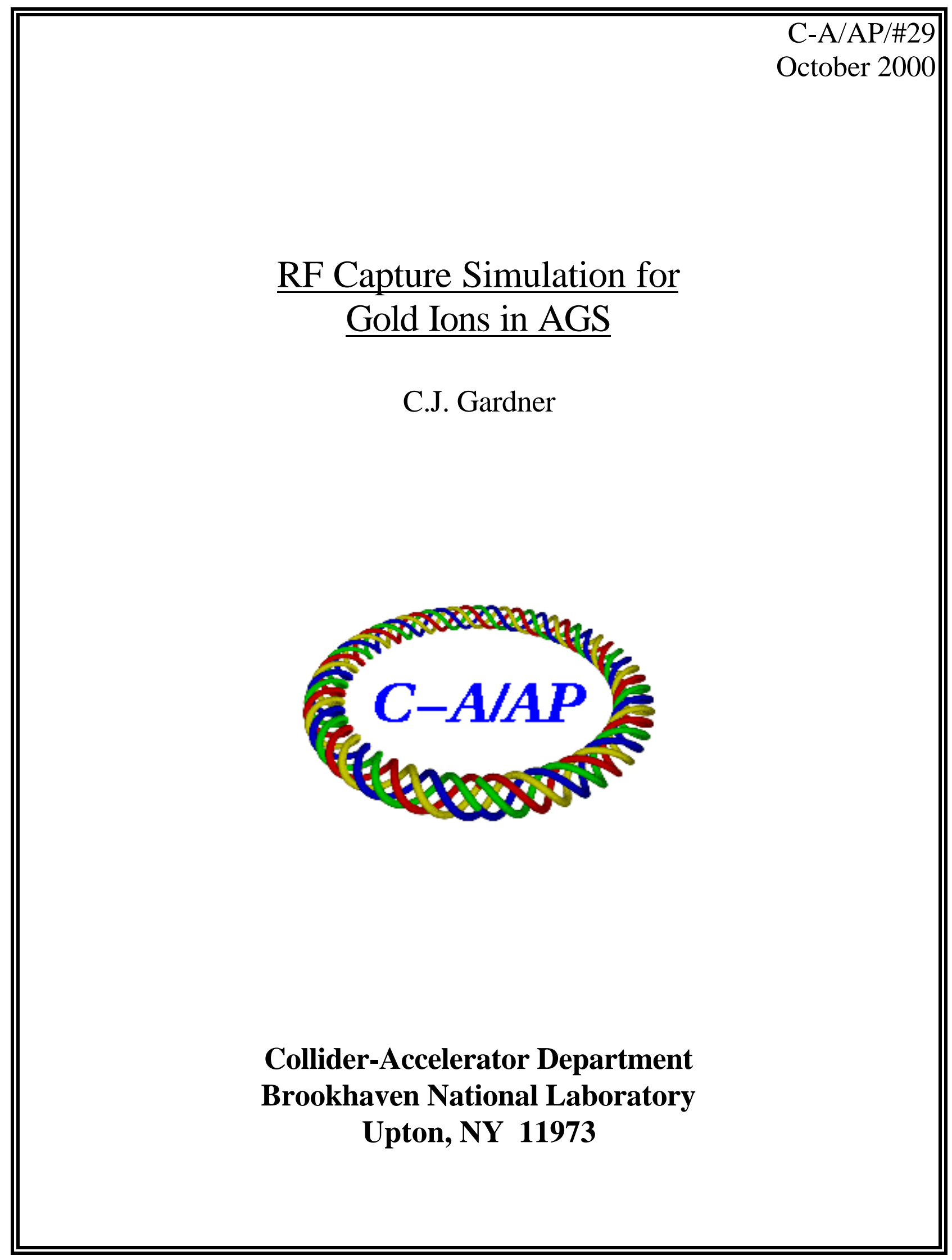




\title{
RF Capture Simulation for Gold Ions in AGS
}

\author{
C.J. Gardner
}

October 31, 2000

Under the present setup for RHIC operation, four batches of six bunches of gold ions $\left(\mathrm{Au}^{77+}\right)$ are injected into the AGS at constant magnetic field on the injection porch of each AGS magnetic cycle. The bunches are injected into stationary RF buckets at harmonic 24 with the $\mathrm{RF}$ voltage adjusted to match the buckets to the bunches as closely as possible. (Due to the large energy spread of the ions emerging from the stripping foil in the BTA line, the required voltage is close to the maximum available - some $320 \mathrm{kV}$.) Shortly after the four batches have been injected, the RF voltage is slowly reduced to zero, adiabatically debunching the beam. This is done because the 24 bunches need to be merged into 4 bunches to meet the luminosity requirements of RHIC. Debunching adiabatically prevents filamentation of the longitudinal emittance (i.e. preserves the "gross emittance") and reduces the energy spread of the beam.

Once debunched and while still on the injection porch, the beam is adiabatically rebunched at harmonic 4 . This is done with a single RF cavity, the so-called KEK cavity, which is the only cavity that can operate at the required low frequency. The other AGS cavities operate at harmonic 12 and are used to accelerate the beam from injection to full energy. Once the beam has been bunched at harmonic 4, the amplitude of this harmonic is slowly decreased to zero and at the same time harmonic 8 is brought on (in the KEK cavity) with every other harmonic 8 bucket centered on a harmonic 4 bucket. As the harmonic 8 and 4 amplitudes are respectively increased and decreased adiabatically, the bunch widths are reduced and the four bunches are captured into every other harmonic 8 bucket. This makes it possible for each bunch to fit inside a harmonic 12 bucket when these are brought on. Finally, bringing on harmonic 12, one ends up with beam captured in every third of 12 stationary buckets on the injection porch. 
This capture scheme was conceived by J. M. Brennan and was successfully implemented by the RF group for the FY2000 RHIC Run. In this note we examine the scheme by numerical simulation.

\section{Turn-by-Turn Equations of Motion}

Calculation of the evolution of a given particle distribution during capture into stationary buckets at constant magnetic field requires a suitable set of turn-by-turn equations for the longitudinal motion. These are derived here following the treatment of MacLachlan [1].

\subsection{Synchronous Parameters}

Let $2 \pi R$ and $\rho$ be the circumference and radius-of-curvature of the design orbit in a given ring, and let $B$ and $2 \pi R_{s}$ be the magnetic field and orbit circumference for the synchronous particle. We assume that $B$ and $R_{s}$ are given and calculate the other synchronous particle parameters in terms of these. Thus the radius-of-curvature is

$$
\rho_{s}=\rho\left(R_{s} / R\right)^{1 / \alpha}, \quad \alpha=\frac{1}{\gamma_{t}^{2}}
$$

and the momentum and energy are

$$
c p_{s}=e Q B \rho_{s}, \quad E_{s}=\sqrt{\left(c p_{s}\right)^{2}+m^{2} c^{4}} .
$$

Here $\alpha$ is the "momentum compaction" factor, $\gamma_{t}$ is the transition gamma, $e$ is the proton charge, and $e Q$ and $m$ are the charge and mass of the particle. The synchronous $\beta, \gamma$ and angular velocity are

$$
\beta_{s}=c p_{s} / E_{s}, \quad \gamma_{s}=E_{s} /\left(m c^{2}\right), \quad \omega_{s}=c \beta_{s} / R_{s} .
$$

We also define the phase slip factor

$$
\eta_{s}=\alpha-\frac{1}{\gamma_{s}^{2}}=\frac{1}{\gamma_{t}^{2}}-\frac{1}{\gamma_{s}^{2}}
$$

\subsection{Time Equation}

We consider a ring with a single RF gap. Let $T_{n}^{s}$ and $T_{n}$ be respectively the times at which the synchronous and asynchronous particles make their 
$n$th pass through the gap. The synchronous particle experiences no acceleration in the gap and revolves around the ring with constant angular velocity $\omega_{s}$. Thus

$$
T_{n+1}^{s}=T_{n}^{s}+T_{s}, \quad T_{s}=2 \pi / \omega_{s},
$$

and taking $T_{1}^{s}=0$, it follows that

$$
T_{n+1}^{s}=n T_{s} .
$$

Similarly, for the asynchronous particle we have

$$
T_{n+1}=T_{n}+2 \pi / \omega_{n}
$$

where $\omega_{n}$ is the angular velocity just after the $n$th pass through the gap. Defining

$$
t_{n}=T_{n}-T_{n}^{s}, \quad t_{n+1}=T_{n+1}-T_{n+1}^{s}
$$

we then have

$$
t_{n+1}=t_{n}+2 \pi\left(\frac{1}{\omega_{n}}-\frac{1}{\omega_{s}}\right)=t_{n}+\left(\frac{\omega_{s}-\omega_{n}}{\omega_{n}}\right) T_{s}
$$

\subsection{Energy Equation}

Now let $E_{n}$ be the energy of the asynchronous particle just after its $n$th pass through the gap. Then we have

$$
E_{n+1}=E_{n}+e Q V\left(T_{n+1}\right),
$$

where $V(T)$ is the voltage across the the gap at time $T$. Since the synchronous particle undergoes no acceleration we must have

$$
V\left(T_{n}^{s}\right)=0
$$

for all $n$. We shall also assume that

$$
V\left(T+T_{s} / h\right)=V(T)
$$

where $h$, the harmonic number, is a positive integer. In terms of $E_{n}$, the other asynchronous parameters are

$$
c p_{n}=\sqrt{E_{n}^{2}-m^{2} c^{4}}, \quad \beta_{n}=c p_{n} / E_{n}
$$

and

$$
\rho_{n}=\frac{c p_{n}}{e Q B}, \quad R_{n}=R\left(\rho_{n} / \rho\right)^{\alpha}, \quad \omega_{n}=c \beta_{n} / R_{n}
$$


Defining

$$
e_{n}=E_{n}-E_{s}, \quad e_{n+1}=E_{n+1}-E_{s}
$$

and using

$$
T_{n+1}=t_{n+1}+T_{n+1}^{s}=t_{n+1}+n T_{s}
$$

and

$$
V\left(t_{n+1}+n T_{s}\right)=V\left(t_{n+1}\right)
$$

we can write (10) as

$$
e_{n+1}=e_{n}+e Q V\left(t_{n+1}\right)
$$

This, together with

$$
t_{n+1}=t_{n}+\left(\frac{\omega_{s}-\omega_{n}}{\omega_{n}}\right) T_{s}
$$

gives the turn-by-turn longitudinal motion of the asynchronous particle.

\subsection{Sympletic Map}

The Jacobian matrix elements for the map from $\left(t_{n}, e_{n}\right)$ to $\left(t_{n+1}, e_{n+1}\right)$ are

$$
\begin{gathered}
\frac{\partial t_{n+1}}{\partial t_{n}}=1, \quad \frac{\partial t_{n+1}}{\partial e_{n}}=2 \pi \frac{\partial\left(1 / \omega_{n}\right)}{\partial e_{n}} \\
\frac{\partial e_{n+1}}{\partial t_{n}}=e Q V^{\prime}, \quad \frac{\partial e_{n+1}}{\partial e_{n}}=1+2 \pi e Q V^{\prime} \frac{\partial\left(1 / \omega_{n}\right)}{\partial e_{n}}
\end{gathered}
$$

where $V^{\prime}$ is the derivative of $V$ with respect to $T$ at time $T_{n+1}$. Thus we have

$$
\left(\frac{\partial t_{n+1}}{\partial t_{n}}\right)\left(\frac{\partial e_{n+1}}{\partial e_{n}}\right)-\left(\frac{\partial t_{n+1}}{\partial e_{n}}\right)\left(\frac{\partial e_{n+1}}{\partial t_{n}}\right)=1
$$

and the map is symplectic. If $t_{n}=0$ and $e_{n}=0$, then it follows from (18) and (19) that $t_{n+1}=0$ and $e_{n+1}=0$. Thus if $t_{1}=0$ and $e_{1}=0$ it follows by induction that $t_{n}=0$ and $e_{n}=0$ for all $n$, and we see that the point $\left(t_{1}, e_{1}\right)=(0,0)$ is a fixed point.

\subsection{Approximate Time Equation}

Now

$$
\frac{\omega_{s}}{\omega_{n}}=1-\left(\frac{\omega_{n}-\omega_{s}}{\omega_{s}}\right)+\left(\frac{\omega_{n}-\omega_{s}}{\omega_{s}}\right)^{2}-\left(\frac{\omega_{n}-\omega_{s}}{\omega_{s}}\right)^{3}+\cdots
$$


and, to first order in $p_{n}-p_{s}$ and $E_{n}-E_{s}$,

$$
\left(\frac{\omega_{n}-\omega_{s}}{\omega_{s}}\right)=-\eta_{s}\left(\frac{p_{n}-p_{s}}{p_{s}}\right)=-\eta_{s}\left(\frac{E_{n}-E_{s}}{\beta_{s}^{2} E_{s}}\right) .
$$

Thus, to first order we have

$$
\frac{\omega_{s}}{\omega_{n}}=1-\left(\frac{\omega_{n}-\omega_{s}}{\omega_{s}}\right)=1+\eta_{s}\left(\frac{E_{n}-E_{s}}{\beta_{s}^{2} E_{s}}\right)=1+\left(\frac{\eta_{s}}{\beta_{s}^{2} E_{s}}\right) e_{n}
$$

and equation (19) becomes

$$
t_{n+1}=t_{n}+T_{s}\left(\frac{\eta_{s}}{\beta_{s}^{2} E_{s}}\right) e_{n}
$$

This, together with

$$
e_{n+1}=e_{n}+e Q V\left(t_{n+1}\right)
$$

again produces a symplectic map from $\left(t_{n}, e_{n}\right)$ to $\left(t_{n+1}, e_{n+1}\right)$.

\subsection{Phase Equation}

Let us now introduce new variables

$$
\phi_{n}=h \omega_{s} t_{n}, \quad W_{n}=\frac{e_{n}}{h \omega_{s}} .
$$

Here $\phi_{n}$ is the phase that corresponds to time $t_{n}$, and $W_{n}$ is defined so that the transformation from $\left(t_{n}, e_{n}\right)$ to $\left(\phi_{n}, W_{n}\right)$ is symplectic. In terms of these variables equations (26) and (27) become

$$
\phi_{n+1}=\phi_{n}+T_{s}\left(\frac{h^{2} \omega_{s}^{2} \eta_{s}}{\beta_{s}^{2} E_{s}}\right) W_{n}
$$

and

$$
W_{n+1}=W_{n}+T_{s}\left(\frac{e Q}{2 \pi h}\right) V\left(\frac{\phi_{n+1}}{h \omega_{s}}\right) .
$$

Defining

$$
a=\left(\frac{h^{2} \omega_{s}^{2} \eta_{s}}{\beta_{s}^{2} E_{s}}\right)
$$

and

$$
F\left(\phi_{n+1}\right)=\left(\frac{e Q}{2 \pi h}\right) V\left(\frac{\phi_{n+1}}{h \omega_{s}}\right)
$$


we then have

$$
\phi_{n+1}=\phi_{n}+a T_{s} W_{n}
$$

and

$$
W_{n+1}=W_{n}+T_{s} F\left(\phi_{n+1}\right) .
$$

These equations again generate a sympletic map. Note that since $V(0)=0$ and $V\left(T+T_{s} / h\right)=V\left(T_{s}\right)$ we have

$$
F(0)=0, \quad F(\phi+2 \pi)=F(\phi) .
$$

Since we are interested in the momentum deviation $p_{n}-p_{s}$, it is useful to have an expression for this in terms of $W_{n}$. Using (24) and (28) we find

$$
\left(\frac{p_{n}-p_{s}}{p_{s}}\right)=\left(\frac{E_{n}-E_{s}}{\beta_{s}^{2} E_{s}}\right)=\left(\frac{h \omega_{s}}{\beta_{s}^{2} E_{s}}\right) W_{n} .
$$

\section{Hamiltonian Equations of Motion}

The turn-by-turn motion given by equations (33) and (34) can be approximated by the motion that follows from a Hamiltonian. The Hamiltonian in this case is

$$
H(\phi, W)=\frac{1}{2} a W^{2}+U(\phi)
$$

and the equations of motion are

$$
\begin{gathered}
\dot{\phi}=\frac{d \phi}{d t}=\frac{\partial H}{\partial W}=a W, \\
\dot{W}=\frac{d W}{d t}=-\frac{\partial H}{\partial \phi}=-\frac{\partial U}{\partial \phi}=F(\phi) .
\end{gathered}
$$

First-order symplectic integration [2] of these equations over time $T_{s}$ yields the symplectic map given by (33) and (34).

\subsection{Constants of the Motion}

If $U(\phi)$ has no explicit dependence on the time, then $H(\phi, W)$ is constant for the motion generated by equations (38) and (39). However, it is only approximately constant for the motion generated by (33) and (34). As 
shown in Ref. [2], one can construct approximate constants order-by-order in $T_{s}$. This is done explicitly to order $T_{s}^{2}$ in the Appendix. Here one finds

$$
H\left(\phi_{n+1}, W_{n+1}\right)=H\left(\phi_{n}, W_{n}\right)+O\left(T_{s}^{2}\right)
$$

where $O\left(T_{s}^{2}\right)$ are terms of order $T_{s}^{2}$ and higher. Proceeding further, one finds

$$
G\left(\phi_{n+1}, W_{n+1}\right)=G\left(\phi_{n}, W_{n}\right)+O\left(T_{s}^{3}\right)
$$

where

$$
G(\phi, W)=H(\phi, W)-\frac{1}{2} a T_{s} W F(\phi)
$$

and $O\left(T_{s}^{3}\right)$ are terms of order $T_{s}^{3}$ and higher.

\subsection{Stable and Unstable Fixed Points}

The fixed points, $\left(\phi_{f}, W_{f}\right)$, of the motion satisfy the equations

$$
0=\frac{\partial H}{\partial \phi}=\frac{\partial U}{\partial \phi}=-F(\phi), \quad 0=\frac{\partial H}{\partial W}=a W .
$$

Thus

$$
F\left(\phi_{f}\right)=0, \quad W_{f}=0 .
$$

To determine whether the motion near a fixed point is stable or unstable we must examine the second derivative of $U$ with respect to $\phi$. Let $U_{\phi \phi}$ be the value of the second derivative at the fixed point. Then, when $a<0$

(below transition), the motion near the fixed point will be stable if $U_{\phi \phi}<0$ and unstable if $U_{\phi \phi}>0$. Similarly when $a>0$ (above transition), the motion near the fixed point will be stable if $U_{\phi \phi}>0$ and unstable if $U_{\phi \phi}<0$.

\subsection{The Separatrix}

Let $H_{u}$ be the value of $H$ at an unstable fixed point: $\phi=\phi_{u}, W=0$. Then

$$
H_{u}=U\left(\phi_{u}\right)
$$

and the equation

$$
H(\phi, W)=H_{u}
$$

defines the separatrix. Solving this equation for $W^{2}(\phi)$ we obtain

$$
W^{2}(\phi)=\frac{2}{a}\left\{U\left(\phi_{u}\right)-U(\phi)\right\} .
$$


We also have

$$
\frac{d W^{2}}{d \phi}=-\frac{2}{a} \frac{\partial U}{\partial \phi}, \quad \frac{d^{2} W^{2}}{d^{2} \phi}=-\frac{2}{a} \frac{\partial^{2} U}{\partial \phi^{2}}
$$

from which it follows that $W^{2}(\phi)$ reaches at local maximum at each stable $\phi_{f}$. The area around a stable fixed point and bounded by the separatrix is an RF bucket. The height, $W_{b}$, of the bucket is given by

$$
W_{b}^{2}=\frac{2}{a}\left\{U\left(\phi_{u}\right)-U\left(\phi_{s}\right)\right\}
$$

where $\phi_{s}$ is the stable $\phi_{f}$.

\section{Capture Simulation}

Let us now use equations of Sections 1 and 2 to simulate the capture process on the injection porch in the AGS.

\subsection{AGS and Gold Parameters}

Gold ions are injected into the AGS with $Q=77, m c^{2}=183.434144 \mathrm{GeV}$, and magnetic rigidity $B \rho_{s}=3.721589 \mathrm{Tm}$. We shall take

$$
\rho_{s}=\rho=85.387 \text { meters }, \quad R_{s}=R=4 R_{r} / 19, \quad \gamma_{t}=8.5
$$

where $R_{r}=3833.845 /(2 \pi)$ meters is the nominal RHIC radius. This gives $R=4 R_{r} / 19=128.4580$ meters, which is approximately $5 \mathrm{~mm}$ larger than the nominal AGS radius reported by Bleser [3]. The momentum and energy of the synchronous gold ion are

$$
c p_{s}=e Q B \rho_{s}, \quad E_{s}=\sqrt{\left(c p_{s}\right)^{2}+m^{2} c^{4}}
$$

and $\beta, \gamma$ and the angular velocity are

$$
\beta_{s}=c p_{s} / E_{s}, \quad \gamma_{s}=E_{s} /\left(m c^{2}\right), \quad \omega_{s}=c \beta_{s} / R_{s} .
$$

Putting in numbers we get $B=435.894$ Gauss, $c p_{s} / n=436.087 \mathrm{MeV}$, $E_{s} / n=1028.20 \mathrm{MeV}, \beta_{s}=0.424128, T_{s}=2 \pi / \omega_{s}=6.34780 \mu \mathrm{s}$, and $\gamma_{s}=1.10424$. Here $n=197$ is the number of nucleons in a gold nucleus. Since $\gamma_{s}<\gamma_{t}$, injection occurs below transition and we have $\eta_{s}<0$ and $a<0$. 


\subsection{Initial Particle Distribution}

The initial particle distribution for the simulation is that of completely debunched beam. This is the situation on the injection porch after four batches of six bunches have been injected (into 24 stationary buckets) and debunched adiabatically. We assume a uniform distribution and consider an 80-by-80 array of points (particles) which cover the region

$$
-\pi \leq \phi \leq \pi, \quad-W_{I} \leq W \leq W_{I}
$$

occupied by one fourth of the beam. Here

$$
W_{I}=E_{I} /\left(h \omega_{s}\right)=E_{I} T_{s} /(2 \pi h)
$$

where $2 E_{I}$ is the energy width of the debunched beam and $h=4$. The area of the region is

$$
\epsilon=(2 \pi)\left(2 W_{I}\right)=2 E_{I} T_{s} / h=E_{I} T_{s} / 2 .
$$

This is the total longitudinal emittance of 6 of the original 24 bunches. We shall take $\epsilon=60 \mathrm{eV}$-s which amounts to $60 / 197=0.305 \mathrm{eV}$-s per nucleon. Each of the 6400 particles in the 80 -by- 80 array will be tracked using the turn-by-turn equations of Section 1. The equations of Section 2 give the parameters of the RF buckets which contain the particles.

\subsection{Capture Program}

For capture with harmonics 4, 8, and 12 on the AGS injection porch, we take 4 to be the fundamental harmonic, and 8 and 12 to be the second and third harmonics of the fundamental. Thus we put $h=4$ in the turn-by-turn equations of Section 1 and we put

$$
F(\phi)=A_{1} \sin \phi+A_{2} \sin 2 \phi+A_{3} \sin 3 \phi
$$

for $F(\phi)$ in the equations of Sections 1 and 2. Here

$$
A_{i}=\left(\frac{e Q V_{i}}{2 \pi h}\right)
$$

and $V_{1}, V_{2}, V_{3}$ are respectively the $\mathrm{RF}$ voltages for harmonics 4,8 , and 12 . These are varied slowly (adiabatically) during the capture process. 


\subsubsection{Harmonic 4 Excitation}

The capture process begins with the excitation of the KEK cavity at harmonic 4. $A_{2}$ and $A_{3}$ are zero and $A_{1}$ is brought up slowly from zero to $A_{K}$ over time $T_{1}$. For $0 \leq T \leq T_{1}$, we have

$$
F(\phi)=A_{1} \sin \phi, \quad U(\phi)=A_{1} \cos \phi
$$

where

$$
A_{1}(T)=A_{K}\left(T / T_{1}\right)^{2} .
$$

Here the amplitude increases quadratically with time as is done in practice [4]. The unstable phases for the bucket centered on $\phi=0$ are $\phi= \pm \pi$, and the bucket separatrix is given by

$$
W^{2}(\phi)=\frac{2}{a}\{U(\pi)-U(\phi)\}=-\frac{2 A_{1}}{a}\{1+\cos \phi\} .
$$

Using the identity $1+\cos \phi=2 \cos ^{2}(\phi / 2)$, this becomes

$$
W^{2}(\phi)=-\frac{4 A_{1}}{a} \cos ^{2}(\phi / 2) \text {. }
$$

The bucket height, $W_{1}$, is given by

$$
W_{1}^{2}=\frac{2}{a}\{U(\pi)-U(0)\}=-\frac{4 A_{1}}{a}=\left(\frac{-2 e Q V_{1} \beta_{s}^{2} E_{s}}{\pi h^{3} \omega_{s}^{2} \eta_{s}}\right)
$$

and the (single) bucket area is

$$
B_{1}=2 \int_{-\pi}^{\pi} W(\phi) d \phi=2 W_{1} \int_{-\pi}^{\pi} \cos (\phi / 2) d \phi=8 W_{1} .
$$

Equating the unbunched beam emittance $(\epsilon=60 \mathrm{eV}$-s $)$ with $B_{1}$, and solving for $V_{1}$, we obtain the minimum voltage required to capture the beam. Thus

$$
\epsilon^{2}=B_{1}^{2}=64 W_{1}^{2}=\left(\frac{-128 e Q V_{1} \beta_{s}^{2} E_{s}}{\pi h^{3} \omega_{s}^{2} \eta_{s}}\right)
$$

and

$$
e V_{1}=\left(\frac{-\epsilon^{2} \pi h^{3} \omega_{s}^{2} \eta_{s}}{128 Q \beta_{s}^{2} E_{s}}\right)=1.6 \mathrm{keV}
$$

Of course, the actual voltage used will be much larger so that the captured beam ends up occupying the central region of a much larger bucket. The maximum voltage available in the KEK cavity is $V_{K}=20 \mathrm{kV}$. Setting $V_{1}=V_{K}$ gives a maximum harmonic 4 bucket area $B_{1}=213 \mathrm{eV}-\mathrm{s}$ (which amounts to $213 / 197=1.08 \mathrm{eV}$-s per nucleon). 


\subsubsection{Harmonic 4 and 8 Excitation}

Capture continues with KEK cavity excitation at both harmonics 4 and 8 . $A_{1}$ is slowly reduced to zero as $A_{2}$ is slowly increased from zero to $A_{K}$ over time $T_{2}-T_{1}$. For $T_{1} \leq T \leq T_{2}$, we have

$$
F(\phi)=A_{1} \sin \phi+A_{2} \sin 2 \phi=\left\{A_{1}+2 A_{2} \cos \phi\right\} \sin \phi,
$$

and

$$
U(\phi)=A_{1} \cos \phi+\frac{1}{2} A_{2}\{\cos 2 \phi+1\}=A_{1} \cos \phi+A_{2} \cos ^{2} \phi
$$

where

$$
A_{1}(T)=A_{K}\left(\frac{T_{2}-T}{T_{2}-T_{1}}\right), \quad A_{2}(T)=A_{K}\left(\frac{T-T_{1}}{T_{2}-T_{1}}\right) .
$$

Here the harmonic 4 amplitude decreases linearly from $A_{K}$ at time $T_{1}$ to zero at time $T_{2}$. Similarly, the harmonic 8 amplitude increases linearly from zero at time $T_{1}$ to $A_{K}$ at time $T_{2}$. This is approximately what is done in practice [4]. Initially, $2 A_{2}<A_{1}$ and the unstable phases for the bucket centered on $\phi=0$ are $\phi_{u}= \pm \pi$. However, as soon as $2 A_{2}$ becomes greater than $A_{1}$, these phases become stable and the unstable ones are given by

$$
A_{1}+2 A_{2} \cos \phi_{u}=0 .
$$

These move toward $\phi_{u}= \pm \pi / 2$ as $A_{1}$ goes to zero. At time $T_{2}$, we have $A_{1}=0, A_{2}=A_{K}$, and only harmonic 8 is excited. The bucket separatrix is then given by

$$
W^{2}(\phi)=\frac{2}{a}\{U(\pi / 2)-U(\phi)\}=-\frac{2 A_{2}}{a} \cos ^{2} \phi
$$

and the bucket height, $W_{2}$, is given by

$$
W_{2}^{2}=\frac{2}{a}\{U(\pi / 2)-U(0)\}=-\frac{2 A_{2}}{a}=\left(\frac{-e Q V_{2} \beta_{s}^{2} E_{s}}{\pi h^{3} \omega_{s}^{2} \eta_{s}}\right) .
$$

Thus we can write

$$
W^{2}(\phi)=W_{2}^{2} \cos ^{2} \phi
$$

and the (single) bucket area is

$$
B_{2}=2 \int_{-\pi / 2}^{\pi / 2} W(\phi) d \phi=2 W_{2} \int_{-\pi / 2}^{\pi / 2} \cos \phi d \phi=4 W_{2} .
$$

Setting $V_{2}=V_{K}=20 \mathrm{kV}$ gives $B_{2}=75 \mathrm{eV}$-s, the largest harmonic 8 bucket area available with the KEK cavity; this is not much larger than the $60 \mathrm{eV}$-s emittance we have assumed. 


\subsubsection{Harmonic 8 and 12 Excitation}

Once the beam has been captured into every other bucket at harmonic 8 , capture into every third bucket at harmonic 12 begins. $A_{2}$ is slowly reduced to zero as $A_{3}$ is slowly increased from zero to $A$ over time $T_{3}-T_{2}$. For $T_{2} \leq T \leq T_{3}$, we have

$$
\begin{aligned}
F(\phi) & =A_{2} \sin 2 \phi+A_{3} \sin 3 \phi \\
& =2 A_{2} \cos \phi \sin \phi+A_{3}\left\{3 \sin \phi-4 \sin ^{3} \phi\right\} \\
& =\left\{2 A_{2} \cos \phi+A_{3}\left(4 \cos ^{2} \phi-1\right)\right\} \sin \phi
\end{aligned}
$$

and

$$
\begin{aligned}
U(\phi) & =\frac{1}{2} A_{2}\{\cos 2 \phi+1\}+\frac{1}{3} A_{3} \cos 3 \phi \\
& =A_{2} \cos ^{2} \phi+\frac{1}{3} A_{3}\left\{4 \cos ^{3} \phi-3 \cos \phi\right\}
\end{aligned}
$$

where

$$
A_{2}(T)=A_{K}\left(\frac{T_{3}-T}{T_{3}-T_{2}}\right), \quad A_{3}(T)=A\left(\frac{T-T_{2}}{T_{3}-T_{2}}\right) .
$$

Here the harmonic 8 amplitude decreases linearly from $A_{K}$ at time $T_{2}$ to zero at time $T_{3}$. Similarly, the harmonic 12 amplitude increases linearly from zero at time $T_{2}$ to $A$ at time $T_{3}$. This, again, is approximately what is done in practice [4]. Solving

$$
2 A_{2} \cos \phi+A_{3}\left(4 \cos ^{2} \phi-1\right)=0
$$

we find that for $A_{3}>0$, the unstable phases closest to $\phi=0$ are given by

$$
\cos \phi_{u}=\frac{-A_{2}+\sqrt{A_{2}^{2}+4 A_{3}^{2}}}{4 A_{3}},
$$

and for $A_{3}>2 A_{2} / 3$, the stable phases closest to $\phi=0$ are given by

$$
\cos \phi_{s}=\frac{-A_{2}-\sqrt{A_{2}^{2}+4 A_{3}^{2}}}{4 A_{3}} .
$$

Thus, as $A_{2}$ goes to zero, the unstable phases move from $\pm \pi / 2$ to $\pm \pi / 3$, and, as soon as $A_{3}>2 A_{2} / 3$, the stable phases begin to move from $\pm \pi$ to 
$\pm 2 \pi / 3$. At time $T_{3}$, we have $A_{2}=0, A_{3}=A$, and only harmonic 12 is excited. The bucket separatrix is then given by

$$
W^{2}(\phi)=\frac{2}{a}\{U(\pi / 3)-U(\phi)\}=-\frac{2 A_{3}}{3 a}\{1+\cos 3 \phi\}
$$

and the bucket height, $W_{3}$, is given by

$$
W_{3}^{2}=\frac{2}{a}\{U(\pi / 3)-U(0)\}=-\frac{4 A_{3}}{3 a}=\left(\frac{-2 e Q V_{3} \beta_{s}^{2} E_{s}}{3 \pi h^{3} \omega_{s}^{2} \eta_{s}}\right) .
$$

Thus we can write

$$
W^{2}(\psi / 3)=\frac{1}{2} W_{3}^{2}\{1+\cos \psi\}=W_{3}^{2} \cos ^{2}(\psi / 2)
$$

and the (single) bucket area is

$$
B_{3}=2 \int_{-\pi / 3}^{\pi / 3} W(\phi) d \phi=\frac{2}{3} \int_{-\pi}^{\pi} W(\psi / 3) d \psi=\frac{8 W_{3}}{3} .
$$

Setting $B_{3}=\epsilon=60 \mathrm{eV}$-s and solving (81) for $V_{3}$, we find

$$
e V_{3}=\left(\frac{-27 \epsilon^{2} \pi h^{3} \omega_{s}^{2} \eta_{s}}{128 Q \beta_{s}^{2} E_{s}}\right)=43 \mathrm{keV}
$$

This is the minimum harmonic 12 voltage required to contain the $60 \mathrm{eV}-\mathrm{s}$ emittance we have assumed.

\section{Results}

The simulation was run with $T_{1}=100 \mathrm{~ms}, T_{2}=150 \mathrm{~ms}, T_{3}=200 \mathrm{~ms}$, and with

$$
A_{K}=\left(\frac{e Q V_{K}}{2 \pi h}\right), \quad A=\left(\frac{e Q V_{A}}{2 \pi h}\right)
$$

where $V_{K}=20 \mathrm{kV}$ and $V_{A}=60 \mathrm{kV}$.

1. Figure 1 shows the beam distribution in the harmonic 4 bucket at time $T_{1}$. Here the rectangle outlines the region of the initial $60 \mathrm{eV}-\mathrm{s}$ uniform distribution. The harmonic 4 voltage at time $T_{1}$ is $V_{1}=V_{K}=20 \mathrm{kV}$ which gives bucket area $B_{1}=213 \mathrm{eV}$-s. This is the largest harmonic 4 bucket area available. 


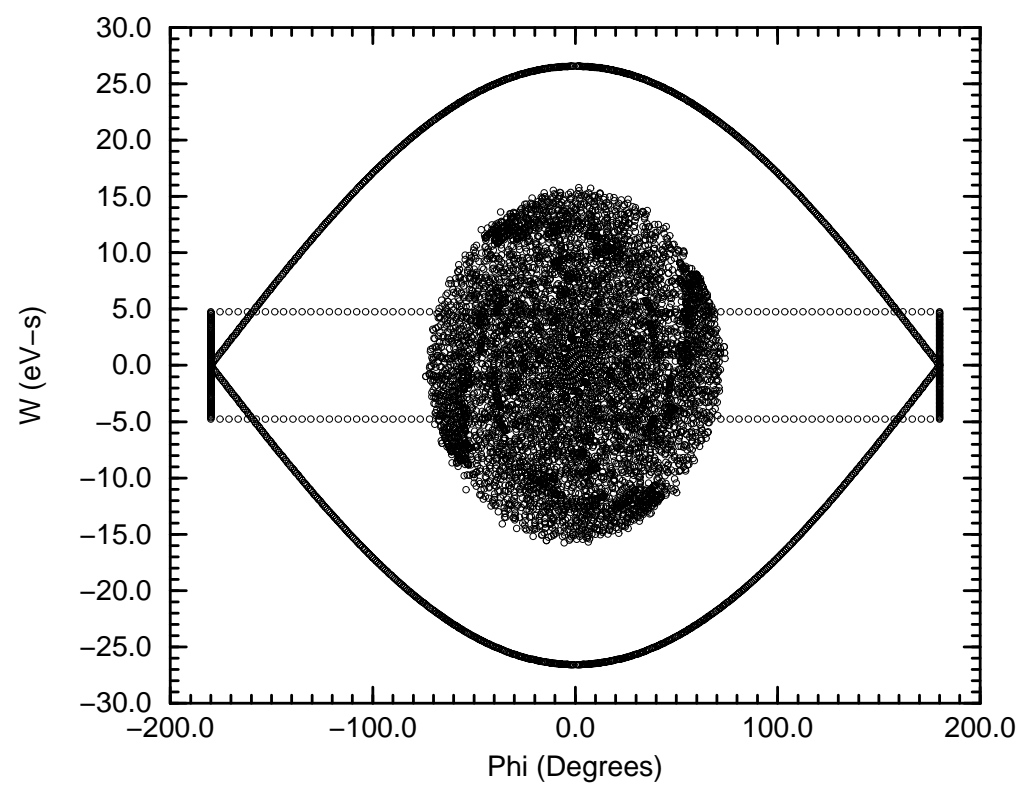

Figure 1: Capture into Harmonic 4 Bucket

2. Figure 2 shows the beam distribution in the harmonic 8 bucket at time $T_{2}$. (The original harmonic 4 bucket at time $T_{1}$ is also shown.) Here the harmonic 8 voltage is $V_{2}=V_{K}=20 \mathrm{kV}$ which gives bucket area $B_{2}=75 \mathrm{eV}$-s. This is the largest harmonic 8 bucket area available and is barely large enough to contain the $60 \mathrm{eV}-\mathrm{s}$ beam emittance.

3. Figure 3 shows the beam distribution in the harmonic 12 bucket at time $T_{3}$. Here the harmonic 12 voltage is $V_{3}=V_{A}=60 \mathrm{kV}$ which gives bucket area $B_{3}=71 \mathrm{eV}$-s. The bucket height is equal to that of the original harmonic 4 bucket. Although the bucket is large enough to contain the $60 \mathrm{eV}$-s emittance, a few particles have managed to leak into the adjacent buckets.

4. Figures 4, 5, 6 show the momentum distributions in the harmonic 4, 8,12 buckets at times $T_{1}, T_{2}, T_{3}$ respectively. Here the time and fractional momentum deviation $d p / p_{s}$ are given by equations (28) and (36). 


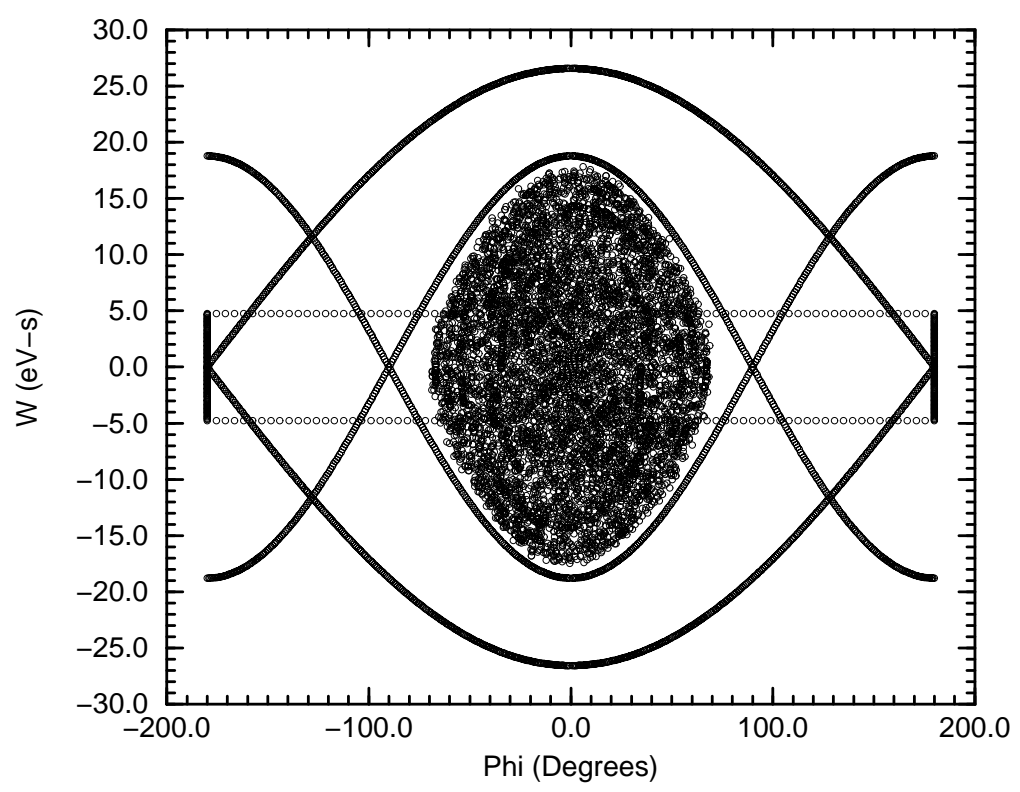

Figure 2: Capture into Harmonic 8 Bucket

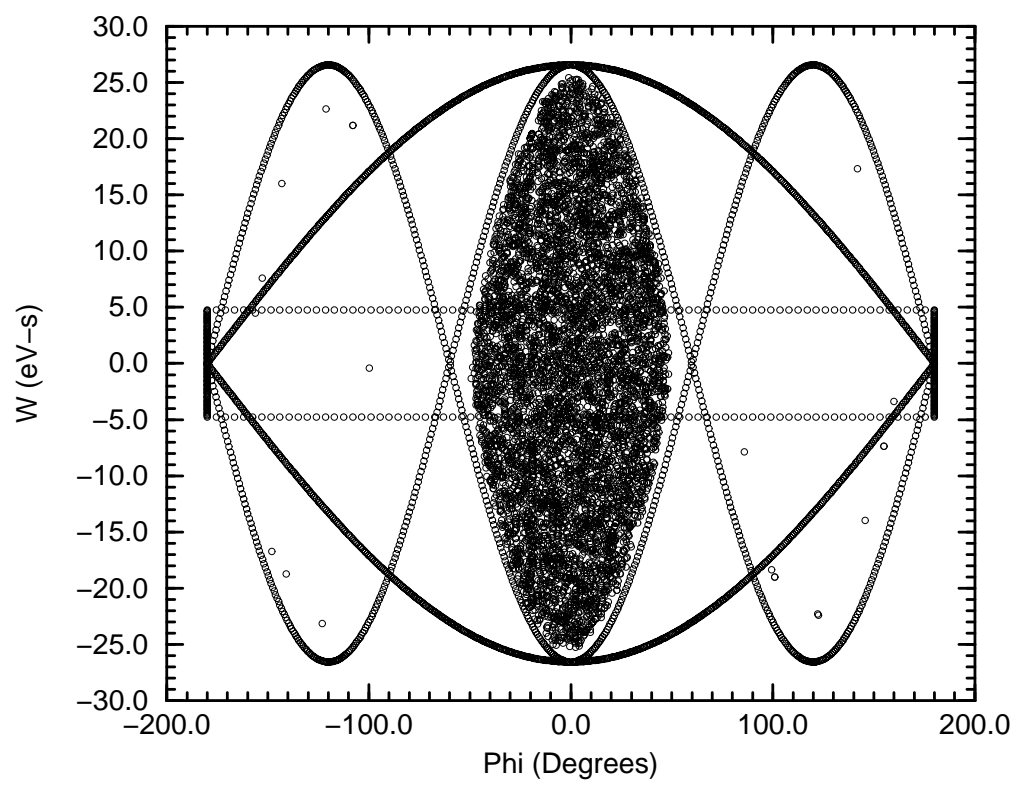

Figure 3: Capture into Harmonic 12 Bucket 


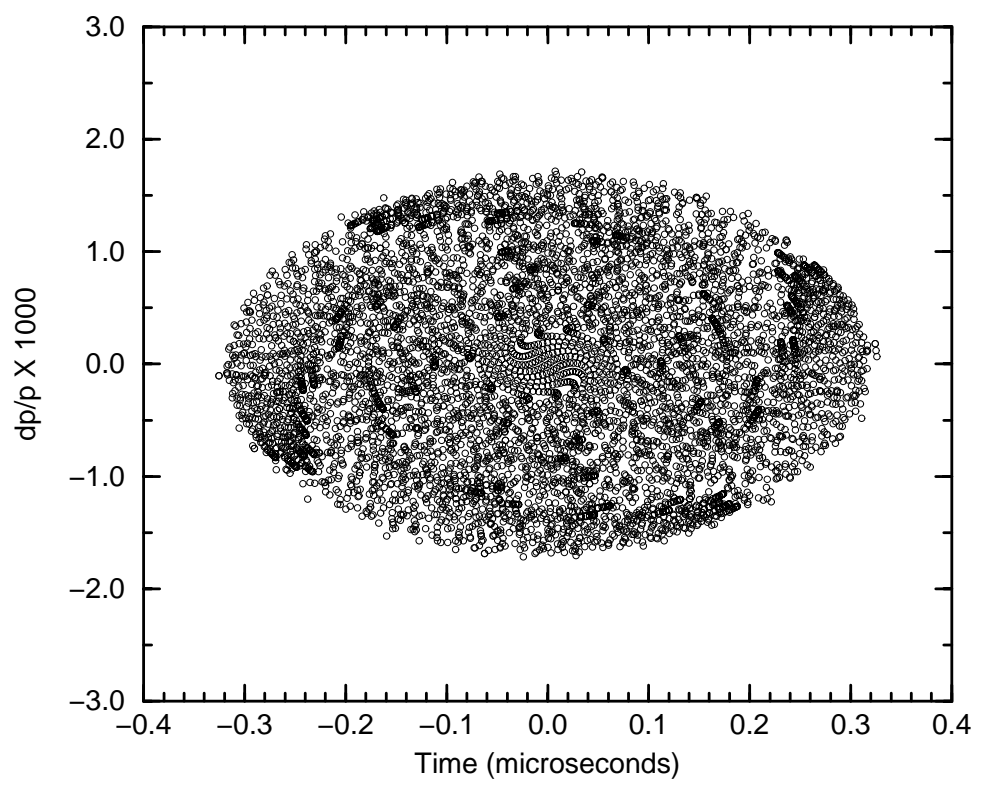

Figure 4: Momentum Distribution in Harmonic 4 Bucket

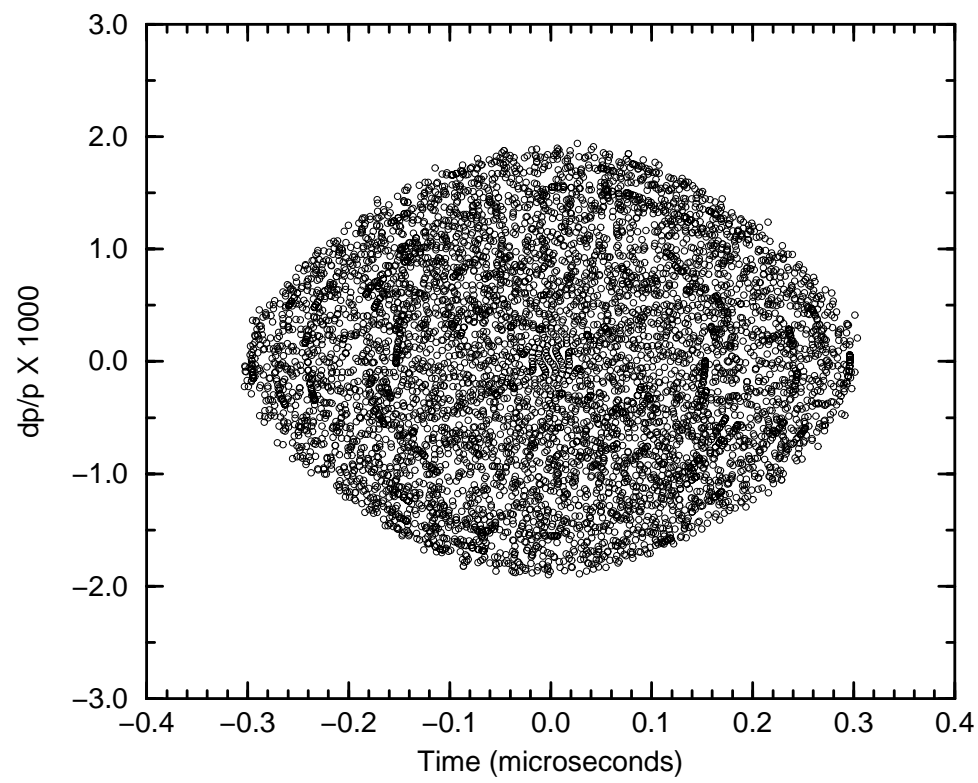

Figure 5: Momentum Distribution in Harmonic 8 Bucket 


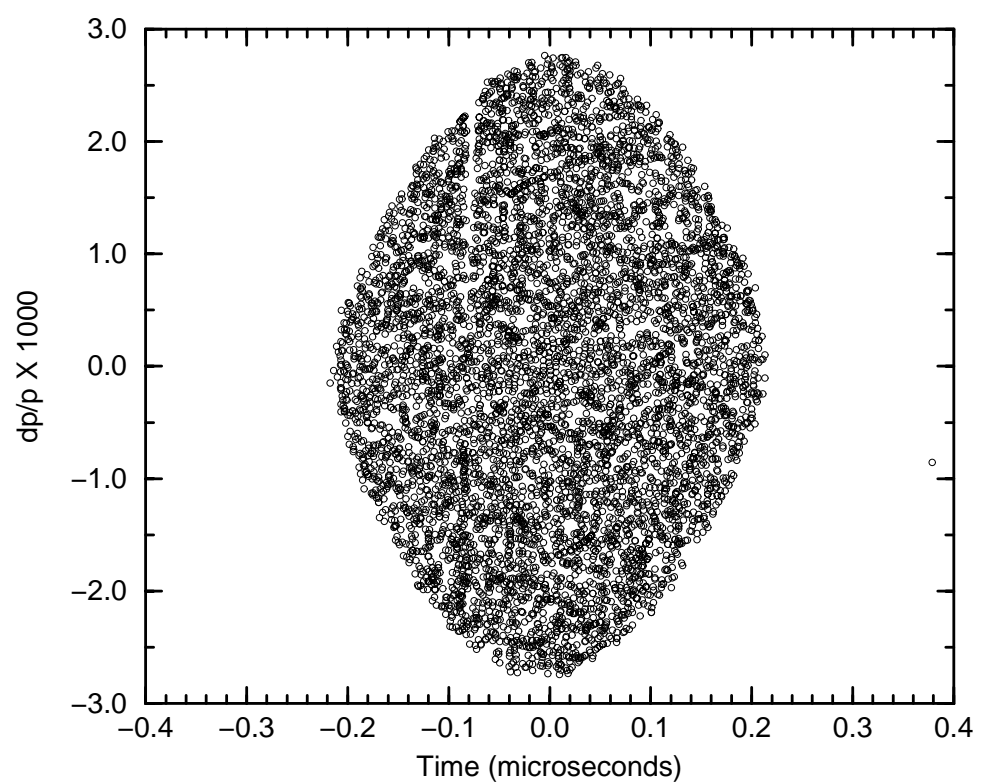

Figure 6: Momentum Distribution in Harmonic 12 Bucket

The "bottleneck" in the capture process is the harmonic 8 bucket area. Because this area is barely large enough to contain the $60 \mathrm{eV}$-s emittance, some particles can end up close to the unstable fixed points as the harmonic 8 voltage is decreased and the harmonic 12 voltage is increased. These presumably are the particles that have leaked into the adjacent harmonic 12 buckets in Figure 3. Increasing the maximum harmonic 12 voltage $V_{A}$ from 60 to $120 \mathrm{kV}$ and increasing the capture time $T_{3}$ from 200 to $250 \mathrm{~ms}$, all but eliminates this leakage.

The simulation was also run with shorter capture times. With $T_{1}=50 \mathrm{~ms}$, $T_{2}=65 \mathrm{~ms}$, and $T_{3}=80 \mathrm{~ms}$, one sees a little more leakage into the adjacent harmonic 12 buckets as shown in Figure 7 . These capture times are comparable to those used in practice [4]. 


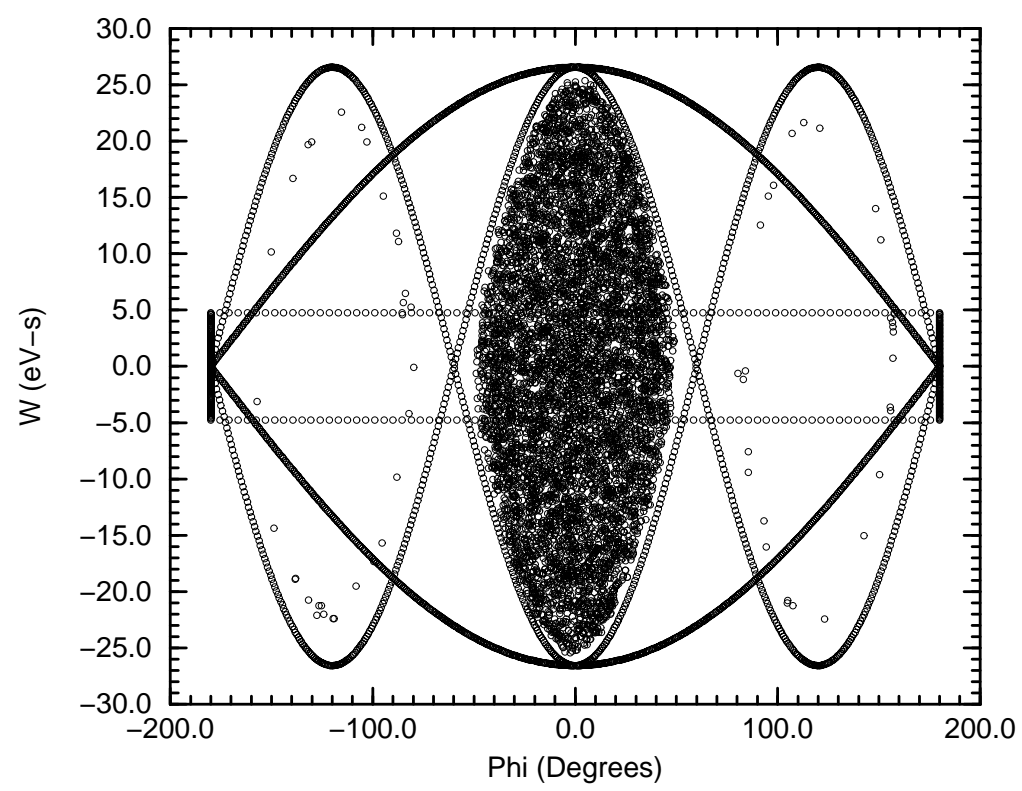

Figure 7: Harmonic 12 Buckets with Shorter Capture Time

\section{$5 \quad$ Appendix}

Consider the general Hamiltonian

$$
H(\phi, W)=T(W)+U(\phi) .
$$

We then have the equations of motion

$$
\begin{gathered}
\frac{d \phi}{d t}=\frac{\partial H}{\partial W}=\frac{d T}{d W}=T^{\prime}=g(W), \\
\frac{d W}{d t}=-\frac{\partial H}{\partial \phi}=-\frac{d U}{d \phi}=-U^{\prime}=f(\phi) .
\end{gathered}
$$

The motion may be approximated by the symplectic map

$$
\phi_{1}=\phi+\tau g(W), \quad W_{1}=W+\tau f\left(\phi_{1}\right) .
$$

We wish to find an approximate constant of the motion generated by this map. This can be done order-by-order in $\tau$ as discussed in Ref. [2]. Let

$$
G(\phi, W)=H_{0}(\phi, W)+\tau H_{1}(\phi, W)
$$


where $H_{0}$ and $H_{1}$ are functions to be determined. To first order in $\tau$ we have

$$
\begin{gathered}
H_{0}\left(\phi_{1}, W_{1}\right)=H_{0}(\phi, W)+\tau g(W) \frac{\partial H_{0}}{\partial \phi}+\tau f(\phi) \frac{\partial H_{0}}{\partial W}, \\
\tau H_{1}\left(\phi_{1}, W_{1}\right)=\tau H_{1}(\phi, W)
\end{gathered}
$$

and

$$
G\left(\phi_{1}, W_{1}\right)=G(\phi, W)+\tau g(W) \frac{\partial H_{0}}{\partial \phi}+\tau f(\phi) \frac{\partial H_{0}}{\partial W} .
$$

If we choose $H_{0}$ such that

$$
\frac{\partial H_{0}}{\partial \phi}=-f(\phi), \quad \frac{\partial H_{0}}{\partial W}=g(W)
$$

then we will have

$$
G\left(\phi_{1}, W_{1}\right)=G(\phi, W)
$$

to first order in $\tau$. Thus we take

$$
H_{0}=T(W)+U(\phi)=H .
$$

Let us now calculate $G\left(\phi_{1}, W_{1}\right)$ to second order in $\tau$. We have

$$
G\left(\phi_{1}, W_{1}\right)=T\left(W_{1}\right)+U\left(\phi_{1}\right)+\tau H_{1}\left(\phi_{1}, W_{1}\right)
$$

where

$$
\begin{gathered}
T\left(W_{1}\right)=T(W)+T^{\prime}(W)\left(W_{1}-W\right)+\frac{1}{2} T^{\prime \prime}\left(W_{1}-W\right)^{2}, \\
U\left(\phi_{1}\right)=U(\phi)+U^{\prime}(\phi)\left(\phi_{1}-\phi\right)+\frac{1}{2} U^{\prime \prime}\left(\phi_{1}-\phi\right)^{2}, \\
T^{\prime}=g(W), \quad T^{\prime \prime}(W)=g^{\prime}(W), \quad U^{\prime}=-f(\phi), \quad U^{\prime \prime}(\phi)=-f^{\prime}(\phi)
\end{gathered}
$$

and

$$
\tau H_{1}\left(\phi_{1}, W_{1}\right)=\tau H_{1}(\phi, W)+\tau\left(\phi_{1}-\phi\right) \frac{\partial H_{1}}{\partial \phi}+\tau\left(W_{1}-W\right) \frac{\partial H_{1}}{\partial W} .
$$

Now

$$
W_{1}-W=\tau f\left(\phi_{1}\right), \quad \phi_{1}-\phi=\tau g(W)
$$


where

$$
f\left(\phi_{1}\right)=f(\phi)+f^{\prime}(\phi)\left(\phi_{1}-\phi\right)=f(\phi)+f^{\prime}(\phi) \tau g(W) .
$$

Thus, to second order in $\tau$, we have

$$
\begin{gathered}
W_{1}-W=\tau f(\phi)+\tau^{2} f^{\prime}(\phi) g(W) \\
\left(W_{1}-W\right)^{2}=\tau^{2} f^{2}(\phi), \quad\left(\phi_{1}-\phi\right)^{2}=\tau^{2} g^{2}(W) .
\end{gathered}
$$

Collecting terms we have

$$
\begin{gathered}
T\left(W_{1}\right)=T(W)+g(W)\left\{\tau f(\phi)+\tau^{2} f^{\prime}(\phi) g(W)\right\}+\frac{1}{2} g^{\prime}(W) \tau^{2} f^{2}(\phi), \\
U\left(\phi_{1}\right)=U(\phi)-f(\phi) \tau g(W)-\frac{1}{2} f^{\prime}(\phi) \tau^{2} g^{2}(W),
\end{gathered}
$$

and

$$
T\left(W_{1}\right)+U\left(\phi_{1}\right)=T(W)+U(\phi)+\frac{1}{2} \tau^{2} f^{\prime}(\phi) g^{2}(W)+\frac{1}{2} \tau^{2} g^{\prime}(W) f^{2}(\phi) .
$$

We also have

$$
\tau H_{1}\left(\phi_{1}, W_{1}\right)=\tau H_{1}(\phi, W)+\tau^{2} g(W) \frac{\partial H_{1}}{\partial \phi}+\tau^{2} f(\phi) \frac{\partial H_{1}}{\partial W} .
$$

Using (108) and (109) in (97), we then have

$$
\begin{aligned}
G\left(\phi_{1}, W_{1}\right) & =G(\phi, W)+\frac{1}{2} \tau^{2} f^{\prime}(\phi) g^{2}(W)+\frac{1}{2} \tau^{2} g^{\prime}(W) f^{2}(\phi) \\
& +\tau^{2} g(W) \frac{\partial H_{1}}{\partial \phi}+\tau^{2} f(\phi) \frac{\partial H_{1}}{\partial W}
\end{aligned}
$$

Thus, choosing $H_{1}$ such that

$$
\frac{\partial H_{1}}{\partial \phi}=-\frac{1}{2} f^{\prime}(\phi) g(W), \quad \frac{\partial H_{1}}{\partial W}=-\frac{1}{2} f(\phi) g^{\prime}(W)
$$

we see that $G\left(\phi_{1}, W_{1}\right)=G(\phi, W)$ to second order in $\tau$. Taking

$$
H_{1}=-\frac{1}{2} f(\phi) g(W)
$$

gives the desired result. 


\section{References}

[1] J.A. MacLachlan, "Difference Equations for Longitudinal Motion in a Synchrotron", Fermilab internal report FNAL FN-529, December 15, 1989; "Differential Equations for Longitudinal Motion in a Synchrotron", Fermilab internal report FNAL FN-532, January 25, 1990.

[2] H. Yoshida, Numerical Integration Methods, Handbook of Accelerator Physics and Engineering, Edited by A.W. Chao and M. Tigner, World Scientific, 1999, pp. 85-87.

[3] E.J. Bleser, "Where are the AGS Magnets", Accelerator Division Technical Note 215, May 20, 1985.

[4] As set up and documented by K.S. Smith. 